3-1-2008

\title{
A Facile and Efficient Synthesis of Some (6E)- Hydroximino-4-En-3-One Steroids, Steroidal Oximes From Cinachyrella spp. Sponges
}

Jianguo Cui

Guangxi Teachers Education University

Liliang Huang

Guangxi Teachers Education University

Lei Fan

Guangxi Teachers Education University

Aimin Zhou

Cleveland State University, A.ZHOU@csuohio.edu

Follow this and additional works at: https://engagedscholarship.csuohio.edu/scichem_facpub

Part of the Chemistry Commons

How does access to this work benefit you? Let us know!

\section{Recommended Citation}

Cui, Jianguo; Huang, Liliang; Fan, Lei; and Zhou, Aimin, "A Facile and Efficient Synthesis of Some (6E)Hydroximino-4-En-3-One Steroids, Steroidal Oximes From Cinachyrella spp. Sponges" (2008). Chemistry Faculty Publications. 374.

https://engagedscholarship.csuohio.edu/scichem_facpub/374

This Article is brought to you for free and open access by the Chemistry Department at EngagedScholarship@CSU. It has been accepted for inclusion in Chemistry Faculty Publications by an authorized administrator of EngagedScholarship@CSU. For more information, please contact library.es@csuohio.edu. 


\title{
A facile and efficient synthesis of some (6E)-hydroximino-4-en-3-one steroids, steroidal oximes from Cinachyrella spp. sponges
}

\author{
Jianguo Cui , Liliang Huang , Lei Fan , Aimin Zhou
}

\section{Introduction}

A variety of steroids with unusual and interesting structures have been isolated from marine sponges, recently [1-3]. Among these steroidal compounds, marine steroids with oxime groups have been reported rarely. In 1997, two steroidal oximes, (6E)-hydroximino-24- ethylcholest-4-en-3-one (1) and (6E)-hydroximinocholest-4-en-3-one (2), were isolated from Cinachyrella alloclada and C. apion [4]. In 2005, another steroidal oxime, (3E)-hydroximinocholest-4-en-6-one (3), was isolated from Cinachyrella australiensis [5]. These steroids exert interesting biological activities [6]. For example, bioassays showed that compound 3 functions against hepatitis virus in vitro [5] and compound 2 exhibitted a selective cytotoxic activity against several types of cancer cells such as P-388, A-549, HT-29 (IC 50 : $1.25 \mu \mathrm{g} / \mathrm{mL}$ ) and MEL-28 tumor cells (IC $50: 2.5 \mu \mathrm{g} / \mathrm{mL}$ ) [7]. In this paper, a facile and efficient synthetic method for the compound 1, 2 and 4 is reported.

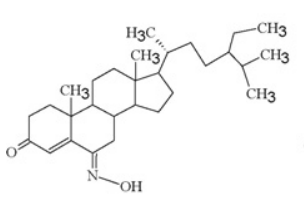

1

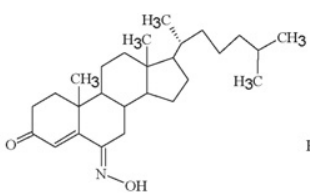

2

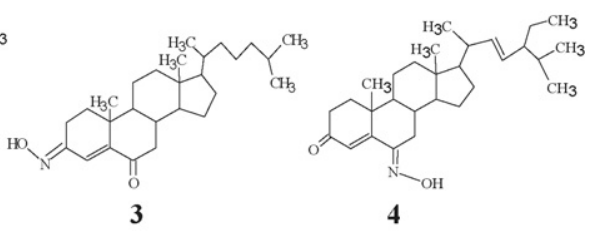




\section{Experimental}

\section{Chemistry}

The sterol and $\mathrm{NaBH}_{4}$ were purchased from the Merck Co. All chemicals and solvents were analytical grade and solvents were purified by general methods before being used. Melting points were determined on an $\mathrm{X}_{4}$ apparatus and were uncorrected. Infrared spectra were measured with a Nicolet FT-360 Spectrophotometer. The ${ }^{1} \mathrm{H}$ and ${ }^{13} \mathrm{CNMR}$ spectra were recorded in $\mathrm{CDCl}_{3}$ on a Bruker AV-500 spectrometer at working frequencies 500 and $125 \mathrm{MHz}$, respectively. Chemical shifts are expressed in ppm $(\delta)$ values and coupling constants $(J)$ in $\mathrm{Hz}$. LREIMS were recorded on a Thermo-DSQ instrument, while HREIMS were measured on a Thermo-MAT95XP instrument. The cell proliferation assay was undertaken by a MTS method using 96-well plates on Beckman coulter LD400 AD/LD analysis spectrometer.

\section{4-Ethylcholest-4-en-3,6-dione (6a)}

Pyridinium chlorochromate (PCC) $(2.564 \mathrm{~g}, 11.9 \mathrm{mmol})$ was added to a solution of sitosterol (5a) $(0.852 \mathrm{~g}, 0.50 \mathrm{mmol})$ in dried $\mathrm{CH}_{2} \mathrm{Cl}_{2}(40 \mathrm{~mL})$ in one portion at room temperature. The reaction was completed in $26 \mathrm{~h}$. To the mixture was then added $30 \mathrm{~mL}$ of $\mathrm{CH}_{2} \mathrm{Cl}_{2}$, and the suspension was poured over a silica gel column and eluted with $\mathrm{CH}_{2} \mathrm{Cl}_{2}$. The resulting solution was washed with cold water and saturated brines. After drying over anhydrous sodium sulfate, the solvent was removed under reduced pressure, and the resulting crude product was purified by chromatography on silica gel using petroleum ether $\left(60-90^{\circ} \mathrm{C}\right) /$ EtOAc $(5: 1)$ as eluent to give $0.75 \mathrm{~g}(86 \%)$ of $6 \mathrm{a}$ as pale yellow crystals, $\theta_{\text {m.p. }} 172-174^{\circ} \mathrm{C}$. IR (KBr) v: 2959 , 1683, 1601, 1581, 1461, 1377, 1246, 1124, 948, $871 \mathrm{~cm}^{-1} ;{ }^{1} \mathrm{H}$ NMR $\left(500 \mathrm{MHz}, \mathrm{CDCl}_{3}\right): 0.724\left(\mathrm{~s}, 3 \mathrm{H}, 18-\mathrm{CH}_{3}\right), 0.816$ (d, 3H, J = 7.0, 26or $\left.27-\mathrm{CH}_{3}\right), 0.841$ (d, 3H, J=7.0, 26- or $\left.27-\mathrm{CH}_{3}\right), 0.848(\mathrm{t}, 3 \mathrm{H}$, $\left.J=8.0,29-\mathrm{CH}_{3}\right), 0.935\left(\mathrm{~d}, 3 \mathrm{H}, \mathrm{J}=6.5,21-\mathrm{CH}_{3}\right), 1.167(\mathrm{~s}, 3 \mathrm{H}, 19-$ $\left.\mathrm{CH}_{3}\right), 2.13-2.17\left(\mathrm{~m}, 1 \mathrm{H}, \mathrm{C}_{2}-\alpha \mathrm{H}\right), 2.44-2.58\left(\mathrm{~m}, 2 \mathrm{H}, \mathrm{C}_{7}-\beta \mathrm{H}\right.$ and $\left.\mathrm{C}_{2}-\beta \mathrm{H}\right), 2.682\left(\mathrm{dd}, 1 \mathrm{H}, J=4.5,15.5, \mathrm{C}_{7}-\alpha \mathrm{H}\right), 6.170\left(\mathrm{~s}, 1 \mathrm{H}, \mathrm{C}_{4}-\mathrm{H}\right)$.

\section{Cholest-4-en-3,6-dione (6b)}

PCC $(2.564 \mathrm{~g}, 11.87 \mathrm{mmol})$ was added to a solution of cholesterol $(0.924 \mathrm{~g}, 2.2 \mathrm{mmol})$ in dried $\mathrm{CH}_{2} \mathrm{Cl}_{2}(40 \mathrm{~mL})$ in one portion at room temperature. The reaction was complete in $28 \mathrm{~h}$. The workup similar to 6 a provided $0.795 \mathrm{~g}(83.5 \%)$ of $6 \mathrm{~b}$ as pale yellow crystals, $\theta_{\text {m.p. }} 90-91^{\circ} \mathrm{C}$; IR(KBr) v: 2953, 2865, 1693, 1600 , 1486, 1249, 1221, 1117, $942 \mathrm{~cm}^{-1} .{ }^{1} \mathrm{H}$ NMR (500 MHz, $\mathrm{CDCl}_{3}$ ): $0.746\left(\mathrm{~s}, 3 \mathrm{H}, 18-\mathrm{CH}_{3}\right), 0.886\left(\mathrm{~d}, 3 \mathrm{H}, \mathrm{J}=6.4,26\right.$ or $\left.27-\mathrm{CH}_{3}\right), 0.899$ (d, $3 \mathrm{H}, \mathrm{J}=6.4,26$ or $\left.27-\mathrm{CH}_{3}\right), 0.952\left(\mathrm{~d}, 3 \mathrm{H}, \mathrm{J}=6.5,21-\mathrm{CH}_{3}\right), 1.172$ (s, 3H, 19- $\mathrm{CH}_{3}$ ), 2.546 (dd, $\left.1 \mathrm{H}, \mathrm{J}=5.2,14.6, \mathrm{C}_{2}-\beta \mathrm{H}\right), 2.706$ (dd, $1 \mathrm{H}$, $\left.J=4.0,16.0, \mathrm{C}_{7}-\alpha \mathrm{H}\right), 6.196\left(\mathrm{~s}, 1 \mathrm{H}, \mathrm{C}_{4}-\mathrm{H}\right)$.

\section{Stigmast-4,22-dien-3,6-dione (6c)}

$6 \mathrm{c}$ was prepared similarly according to the procedure of $6 \mathrm{a}$. PCC (1.30 g, $6.0 \mathrm{mmol})$ was added to a solution of stigmasterol $(0.50 \mathrm{~g}, 1.2 \mathrm{mmol})$ in dried $\mathrm{CH}_{2} \mathrm{Cl}_{2}(10 \mathrm{~mL})$ in one portion at room temperature. The reaction was complete in $27 \mathrm{~h}$. The workup similar to $6 \mathrm{a}$ gave $0.42 \mathrm{~g}(83 \%)$ of $6 \mathrm{c}$ as pale yellow

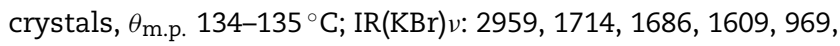
$864 \mathrm{~cm}^{-1} ;{ }^{1} \mathrm{H}$ NMR $\left(500 \mathrm{MHz}, \mathrm{CDCl}_{3}\right): 0.743$ (s, 3H, 18- $\left.\mathrm{CH}_{3}\right), 0.805$ (t, $\left.3 \mathrm{H}, J=7.0,29-\mathrm{CH}_{3}\right), 0.798\left(\mathrm{~d}, 3 \mathrm{H}, J=6.5,26-\right.$ or $\left.27-\mathrm{CH}_{3}\right), 0.849$ $\left(\mathrm{d}, 3 \mathrm{H}, \mathrm{J}=6.5,26\right.$ - or $\left.27-\mathrm{CH}_{3}\right), 1.036\left(\mathrm{~d}, 3 \mathrm{H}, \mathrm{J}=7.0,21-\mathrm{CH}_{3}\right), 1.169$ (s, 3H, 19- $\mathrm{CH}_{3}$ ), 5.040 (dd, $1 \mathrm{H}, J=9.0,15.2, \mathrm{C}_{22}-\mathrm{H}$ ), 5.150 (dd, $1 \mathrm{H}$, $\left.J=8.5,15.2, \mathrm{C}_{23}-\mathrm{H}\right), 6.171\left(\mathrm{~s}, 1 \mathrm{H}, \mathrm{C}_{4}-\mathrm{H}\right)$.

\section{4-Ethylcholest-4-en-3 $\beta$-ol-6-one (7a)}

$\mathrm{NaBH}_{4}(30 \mathrm{mg}, 0.79 \mathrm{mmol})$ was added to a solution of $6 \mathrm{a}$ $(110 \mathrm{mg}, 0.25 \mathrm{mmol})$ and $\mathrm{CoCl}_{2} .6 \mathrm{H}_{2} \mathrm{O}(61 \mathrm{mg}, 0.26 \mathrm{mmol})$ in $\mathrm{CH}_{3} \mathrm{OH}(15 \mathrm{~mL})$ in the interval of $8 \mathrm{~min}$ at room temperature. After $15 \mathrm{~min}$, the reaction was stopped. The solution was neutralized with $1 \mathrm{M} \mathrm{HCl}$. After evaporation of the majority of the $\mathrm{MeOH}$ under reduced pressure, ethyl acetate $(30 \mathrm{~mL})$ was added to the residue. The resulting solution was washed with cold water and saturated brines. After drying over anhydrous sodium sulfate, the solvent was removed under reduced pressure, and the resulting crude product was purified by flash chromatography on silica gel using petroleum ether/ethyl acetate (2:1) as the eluent. The 7a was obtained as a white solid (98 mg, 87\%), $\theta_{\text {m.p. }} 140-141^{\circ} \mathrm{C}$; IR(KBr) v: 3428, 2962, 2929, 2868, 1716, 1659, 1462, 1377, 1074, $968 \mathrm{~cm}^{-1} ;{ }^{1} \mathrm{H} \mathrm{NMR}\left(\mathrm{CDCl}_{3}\right.$, $500 \mathrm{MHz}): 0.735$ (s, 3H, 18- $\left.\mathrm{CH}_{3}\right), 0.839$ (d, 3H, J=7.5, 26- $\mathrm{CH}_{3}$ or $\left.27-\mathrm{CH}_{3}\right), 0.861\left(\mathrm{~d}, 3 \mathrm{H}, \mathrm{J}=7.5,26-\mathrm{CH}_{3}\right.$ or $\left.27-\mathrm{CH}_{3}\right), 0.869(\mathrm{t}, 3 \mathrm{H}$, $\left.J=8.0,29-\mathrm{CH}_{3}\right), 0.943\left(\mathrm{~d}, 3 \mathrm{H}, J=6.0,21-\mathrm{CH}_{3}\right), 1.202(\mathrm{~s}, 3 \mathrm{H}, 19-$ $\left.\mathrm{CH}_{3}\right), 4.342\left(\mathrm{~m}, 1 \mathrm{H}, \mathrm{C}_{3}-\alpha \mathrm{H}\right), 6.193\left(\mathrm{~s}, 1 \mathrm{H}, \mathrm{C}_{4}-\mathrm{CH}\right)$.

\section{Cholest-4-en-3 $\beta$-ol-6-one (7b)}

Yield $88.2 \%, \theta$ m.p. $125-126^{\circ} \mathrm{C}$; IR(KBr) v: 3391, 2946, 2868, 1708, 1663, 1462, 1373, 1274, 1123, 1070, 960, $878 \mathrm{~cm}^{-1} ;{ }^{1} \mathrm{H}$ NMR $\left(\mathrm{CDCl}_{3}, 500 \mathrm{MHz}\right): 0.732$ (s, 3H, 18- $\left.\mathrm{CH}_{3}\right), 0.882$ (d, 3H, J = 6.2, 26$\mathrm{CH}_{3}$ or $\left.27-\mathrm{CH}_{3}\right), 0.895\left(\mathrm{~d}, 3 \mathrm{H}, \mathrm{J}=6.2,26-\mathrm{CH}_{3}\right.$ or $\left.27-\mathrm{CH}_{3}\right), 0.935(\mathrm{~d}$, $\left.3 \mathrm{H}, \mathrm{J}=6.5,21-\mathrm{CH}_{3}\right), 1.184\left(\mathrm{~s}, 3 \mathrm{H}, 19-\mathrm{CH}_{3}\right), 4.306\left(\mathrm{~m}, 1 \mathrm{H}, \mathrm{C}_{3}-\alpha \mathrm{H}\right)$, $6.193\left(\mathrm{~d}, \mathrm{~J}=1.2, \mathrm{C}_{4}-\mathrm{H}\right)$.

\section{4-Ethylcholest-4,22-dien-3 $\beta$-ol-6-one (7c)}

Yield 90\%, $\theta_{\text {m.p. }} 175-176^{\circ} \mathrm{C}$; $\operatorname{IR}(\mathrm{KBr})$ v: 3457, 2954, 2860, 1711, 1659, 1458, 1385, 1279, 1074, $972 \mathrm{~cm}^{-1} ;{ }^{1} \mathrm{H} \mathrm{NMR}\left(\mathrm{CDCl}_{3}\right.$, $500 \mathrm{MHz}$ ): 0.730 (s, 3H, 18- $\left.\mathrm{CH}_{3}\right), 0.797$ (d, 3H, J=6.5, 26- $\mathrm{CH}_{3}$ or $\left.27-\mathrm{CH}_{3}\right), 0.848\left(\mathrm{~d}, 3 \mathrm{H}, \mathrm{J}=6.5,26-\mathrm{CH}_{3}\right.$ or $\left.27-\mathrm{CH}_{3}\right), 0.804(\mathrm{t}, 3 \mathrm{H}$, $\left.J=7.5,29-\mathrm{CH}_{3}\right), 1.019\left(\mathrm{~d}, 3 \mathrm{H}, J=6.5,21-\mathrm{CH}_{3}\right), 1.184(\mathrm{~s}, 3 \mathrm{H}, 19-$ $\mathrm{CH}_{3}$ ), 4.329 (ddd, $1 \mathrm{H}, J=1.5,5.5,12.0, \mathrm{C}_{3}-\alpha \mathrm{H}$ ), 5.026 (dd, $1 \mathrm{H}$, $\left.J=8.5,15.0, C_{22}-H\right), 5.143\left(\mathrm{dd}, 1 \mathrm{H}, J=8.5,15.0, \mathrm{C}_{23}-\mathrm{H}\right), 6.167(\mathrm{~d}$, $\left.1 \mathrm{H}, J=1.5, \mathrm{C}_{4}-\mathrm{H}\right)$.

(6E)-Hydroximino-24-ethylcholest-4-en-3 $\beta$-ol (8a)

7a (150 mg, $0.40 \mathrm{mmol}$ ) was dissolved in $15 \mathrm{~mL} \mathrm{95 \%} \mathrm{CH}_{3} \mathrm{CH}_{2} \mathrm{OH}$. After the mixture was heated to $55^{\circ} \mathrm{C}, \mathrm{CH}_{3} \mathrm{COONa} \cdot 3 \mathrm{H}_{2} \mathrm{O}$ ( $95 \mathrm{mg}, 0.70 \mathrm{mmol}$ ) and $\mathrm{NH}_{2} \mathrm{OH}^{\bullet} \mathrm{HCl}(60.6 \mathrm{mg}, 0.87 \mathrm{mmol})$ were added. The mixture was stirred at the temperature for $1.5 \mathrm{~h}$. Then the reaction was terminated and the majority of solvent was evaporated under reduced pressure. Proper water was added into the reaction mixture, and the product was extracted with ethyl acetate $(3 \mathrm{~mL} \times 20 \mathrm{~mL})$. The combined extracts were washed with saturated brine, dried, and evaporated under reduced pressure. The residue was subjected to chromatography to give $109 \mathrm{mg}$ of 8 a $(70.3 \%), \theta_{\text {m.p. }} 91-92^{\circ} \mathrm{C}$; $\mathrm{IR}(\mathrm{KBr})$ v: 3428, 2958, 2933, 2868, 1634, 1462, $1377 \mathrm{~cm}^{-1} ;{ }^{1} \mathrm{H}$ $\mathrm{NMR}\left(\mathrm{CDCl}_{3}, 500 \mathrm{MHz}\right): 0.722$ (s, 3H, 18- $\left.\mathrm{CH}_{3}\right), 0.839$ (d, 3H, J = 7.0, $26-\mathrm{CH}_{3}$ or $\left.27-\mathrm{CH}_{3}\right), 0.861\left(\mathrm{~d}, 3 \mathrm{H}, \mathrm{J}=7.0,26-\mathrm{CH}_{3}\right.$ or $\left.27-\mathrm{CH}_{3}\right), 0.870$ $\left(\mathrm{t}, 3 \mathrm{H}, \mathrm{J}=8.0,29-\mathrm{CH}_{3}\right), 0.940\left(\mathrm{~d}, 3 \mathrm{H}, \mathrm{J}=6.5,21-\mathrm{CH}_{3}\right), 1.077(\mathrm{~s}, 3 \mathrm{H}$, $\left.19-\mathrm{CH}_{3}\right), 3.064\left(\mathrm{dd}, 1 \mathrm{H}, J=3.0,14.0, \mathrm{C}_{7}-\beta \mathrm{H}\right), 4.284$ (ddd, $1 \mathrm{H}, \mathrm{J}=1.0$, 
3.5, 11.0, $\left.\mathrm{C}_{3}-\alpha \mathrm{H}\right), 6.243$ (s, $\left.1 \mathrm{H}, \mathrm{C}_{4}-\mathrm{H}\right) ;{ }^{13} \mathrm{C} \mathrm{NMR}\left(\mathrm{CDCl}_{3}, 125 \mathrm{MHz}\right)$ : 35.2 (1-C), 29.7 (2-C), 69.1 (3-C), 113.8 (4-C), 156.7 (5-C), 156.9 (6C), 28.1 (7-C), 33.9 (8-C), 53.6 (9-C), 41.7 (10-C), 21.4 (11-C), 39.7 (12-C), 42.5 (13-C), 56.1 (14-C), 23.1 (15-C), 26.2 (16-C), 55.8 (17C), 11.9 (18-C), 19.8 (19-C), 36.1 (20-C), 18.7 (21-C), 34.2 (22-C), 24.1 (23-C), 45.9 (24-C), 29.2 (25-C), 18.4 (26-C), 19.0 (27-C), 24.2 (28-C), 12.0 (29-C).

In the reaction, the $6 Z$-isomer $9 \mathrm{a}$ of $8 \mathrm{a}$ was obtained in $3 \%$ yield, $\theta_{\text {m.p. }} 119-121^{\circ} \mathrm{C}$; IR(KBr) v: 3412, 2958, 2938, 2872, 1630,

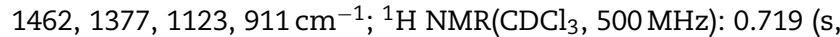
$\left.3 \mathrm{H}, 18-\mathrm{CH}_{3}\right), 0.839\left(\mathrm{~d}, 3 \mathrm{H}, \mathrm{J}=7.0,26-\mathrm{CH}_{3}\right.$ or $\left.27-\mathrm{CH}_{3}\right), 0.861$ (d, $3 \mathrm{H}, \mathrm{J}=7.0,26-\mathrm{CH}_{3}$ or $\left.27-\mathrm{CH}_{3}\right), 0.869\left(\mathrm{t}, 3 \mathrm{H}, \mathrm{J}=8.0,29-\mathrm{CH}_{3}\right), 0.937$ $\left(\mathrm{d}, 3 \mathrm{H}, \mathrm{J}=6.0,21-\mathrm{CH}_{3}\right), 1.109\left(\mathrm{~s}, 3 \mathrm{H}, 19-\mathrm{CH}_{3}\right), 2.333-2.370(\mathrm{~m}, 2 \mathrm{H}$, $\left.\mathrm{C}_{7}-\mathrm{H}\right), 4.322\left(\mathrm{dd}, 1 \mathrm{H}, \mathrm{J}=4.5,12.0, \mathrm{C}_{3}-\alpha \mathrm{H}\right), 6.922\left(\mathrm{~s}, 1 \mathrm{H}, \mathrm{C}_{4}-\mathrm{H}\right) ;{ }^{13} \mathrm{C}$ $\mathrm{NMR}\left(\mathrm{CDCl}_{3}, 125 \mathrm{MHz}\right): 36.1$ (1-C), 29.2 (2-C), 69.3 (3-C), 113.9 (4C), 153.7 (5-C), 160.5 (6-C), 29.7 (7-C), 33.9 (8-C), 53.8 (9-C), 39.6 (10-C), 21.2 (11-C), 39.4 (12-C), 42.0 (13-C), 56.0 (14-C), 24.2 (15C), 26.2 (16-C), 55.7 (17-C), 11.9 (18-C), 19.8 (19-C), 36.8 (20-C), 18.7 (21-C), 34.3 (22-C), 24.5 (23-C), 45.9 (24-C), 28.1 (25-C), 19.0 (26-C), 18.9 (27-C), 23.1 (28-C), 12.0 (29-C).

\section{(6E)-Hydroximinocholest-4-en-3 $\beta$-ol (8b)}

Yield 74.5\%, $\theta_{\text {m.p. }}{ }^{155}-157^{\circ} \mathrm{C}$; IR(KBr) v: 3374, 2949, 2864, 1629, $1462,1376,1315,1119,1070,968,890,727,674 \mathrm{~cm}^{-1} ;{ }^{1} \mathrm{H}$ $\mathrm{NMR}\left(\mathrm{CDCl}_{3}, 500 \mathrm{MHz}\right): 0.717$ (s, 3H, 18- $\left.\mathrm{CH}_{3}\right), 0.882$ (d, 3H, J = 2.0, $26-\mathrm{CH}_{3}$ or $\left.27-\mathrm{CH}_{3}\right), 0.895\left(\mathrm{~d}, 3 \mathrm{H}, \mathrm{J}=2.0,26-\mathrm{CH}_{3}\right.$ or $\left.27-\mathrm{CH}_{3}\right), 0.931$ $\left(\mathrm{d}, 3 \mathrm{H}, J=6.5,21-\mathrm{CH}_{3}\right), 1.070$ (s, 3H, 19- $\mathrm{CH}_{3}$ ), 3.059 (dd, $1 \mathrm{H}, J=3.0$, 14.0, $\mathrm{C}_{7}-\beta \mathrm{H}$ ), $4.273\left(\mathrm{dd}, 1 \mathrm{H}, \mathrm{J}=4.4,12.0 \mathrm{~Hz}, \mathrm{C}_{3}-\alpha \mathrm{H}\right), 6.249(\mathrm{~s}, 1 \mathrm{H}$, $\left.\mathrm{C}_{4}-\mathrm{H}\right)$.

In the reaction, the $6 \mathrm{Z}$-isomer $9 \mathrm{~b}$ of $8 \mathrm{~b}$ was obtained in $4 \%$ yield, $\theta_{\text {m.p. }} 124-126^{\circ} \mathrm{C}$; $\operatorname{IR}(\mathrm{KBr})$ v: $3411,2958,2872,1629$, 1462, $1376 \mathrm{~cm}^{-1} ;{ }^{1} \mathrm{H} \mathrm{NMR}\left(\mathrm{CDCl}_{3}, 500 \mathrm{MHz}\right): 0.762$ (s, 3H, 18$\mathrm{CH}_{3}$ ), 0.894 (d, 3H, J=2.3, 26 or $\left.27-\mathrm{CH}_{3}\right), 0.902(\mathrm{~d}, 3 \mathrm{H}, J=2.3,26$ or 27- $\left.\mathrm{CH}_{3}\right), 0.965\left(\mathrm{~d}, 3 \mathrm{H}, \mathrm{J}=6.5,21-\mathrm{CH}_{3}\right), 1.144\left(\mathrm{~s}, 3 \mathrm{H}, 19-\mathrm{CH}_{3}\right)$, $4.277\left(\mathrm{~m}, 1 \mathrm{H}, \mathrm{C}_{3}-\alpha \mathrm{H}\right), 6.937\left(\mathrm{~d}, 1 \mathrm{H}, J=2.1, \mathrm{C}_{4}-\mathrm{H}\right)$.

\section{(6E)-Hydroximino-24-ethylcholest-4,22-dien-3 $\beta$-ol}

\section{(8c)}

Yield 69.1\%, $\theta_{\text {m.p. }} 96-97^{\circ} \mathrm{C}$; IR(KBr) v: 3399, 2954, 2929, 2868, 1634, 1462, 1381, 1074, $968 \mathrm{~cm}^{-1} ;{ }^{1} \mathrm{H} \mathrm{NMR}\left(\mathrm{CDCl}_{3}, 500 \mathrm{MHz}\right)$ : $0.739\left(\mathrm{~s}, 3 \mathrm{H}, 18-\mathrm{CH}_{3}\right), 0.822\left(\mathrm{~d}, 3 \mathrm{H}, \mathrm{J}=7.7,26-\mathrm{CH}_{3}\right.$ or $\left.27-\mathrm{CH}_{3}\right)$, $0.872\left(\mathrm{~d}, 3 \mathrm{H}, \mathrm{J}=7.0,26-\mathrm{CH}_{3}\right.$ or $\left.27-\mathrm{CH}_{3}\right), 0.829$ (t, $3 \mathrm{H}, J=7.5,29-$ $\left.\mathrm{CH}_{3}\right), 1.040\left(\mathrm{~d}, 3 \mathrm{H}, \mathrm{J}=6.0,21-\mathrm{CH}_{3}\right), 1.078\left(\mathrm{~s}, 3 \mathrm{H}, 19-\mathrm{CH}_{3}\right), 3.064$ (dt, $1 \mathrm{H}, J=3.5,17.5, \mathrm{C}_{7}-\beta \mathrm{H}$ ), 4.282 (ddd, $1 \mathrm{H}, J=2.0,5.5,12.5, \mathrm{C}_{3}$ $\alpha \mathrm{H}), 5.049\left(\mathrm{dd}, 1 \mathrm{H}, \mathrm{J}=9.0,15.0, \mathrm{C}_{22}-\mathrm{H}\right), 5.172(\mathrm{dd}, 1 \mathrm{H}, \mathrm{J}=8.5,15.0$, $\left.\mathrm{C}_{23}-\mathrm{H}\right), 6.247$ (s, $\left.\left.1 \mathrm{H}, \mathrm{C}_{4}-\mathrm{H}\right) ;{ }^{13} \mathrm{C} \mathrm{NMR} \mathrm{CDCl}_{3}, 125 \mathrm{MHz}\right): 35.2(1-\mathrm{C})$, 29.7 (2-C), 69.0 (3-C), 113.9 (4-C), 156.7 (5-C), 156.9 (6-C), 28.8 (7C), 31.9 (8-C), 51.2 (9-C), 39.5 (10-C), 21.2 (11-C), 38.5 (12-C), 40.4 (13-C), 55.9 (14-C), 21.4 (15-C), 25.4 (16-C), 53.6 (17-C), 12.2 (18C), 18.7 (19-C), 41.6 (20-C), 19.0 (21-C), 138.1 (22-C), 129.5 (23-C), 42.4 (24-C), 34.2 (25-C), 21.1 (26-C), 18.4 (27-C), 24.3 (28-C), 12.1 (29-C).

In the reaction, the $6 \mathrm{Z}$-isomer $9 \mathrm{c}$ of $8 \mathrm{c}$ was obtained in $3.5 \%$ yield, $\theta$ m.p. $125-127^{\circ} \mathrm{C}$; IR(KBr): $3420,2958,2868,1638,1462$, 1381, 1025, $976 \mathrm{~cm}^{-1} ;{ }^{1} \mathrm{H} \mathrm{NMR}\left(\mathrm{CDCl}_{3}, 500 \mathrm{MHz}\right): 0.736$ (s, $3 \mathrm{H}$, $\left.18-\mathrm{CH}_{3}\right), 0.820\left(\mathrm{~d}, 3 \mathrm{H}, J=6.0,26-\mathrm{CH}_{3}\right.$ or $\left.27-\mathrm{CH}_{3}\right), 0.826(\mathrm{t}, 3 \mathrm{H}$, $\left.J=8.0,29-\mathrm{CH}_{3}\right), 0.870\left(\mathrm{~d}, 3 \mathrm{H}, \mathrm{J}=6.0,26-\mathrm{CH}_{3}\right.$ or $\left.27-\mathrm{CH}_{3}\right), 1.036(\mathrm{~d}$, $\left.3 \mathrm{H}, \mathrm{J}=6.5,21-\mathrm{CH}_{3}\right), 1.110\left(\mathrm{~s}, 3 \mathrm{H}, 19-\mathrm{CH}_{3}\right), 4.323(\mathrm{dd}, 1 \mathrm{H}, \mathrm{J}=4.0$, 11.5, $\left.\mathrm{C}_{3}-\alpha \mathrm{H}\right), 5.047\left(\mathrm{dd}, 1 \mathrm{H}, \mathrm{J}=9.0,14.5, \mathrm{C}_{22}-\mathrm{H}\right), 5.168(\mathrm{dd}, 1 \mathrm{H}$, $\left.J=8.5,14.5, C_{23}-\mathrm{H}\right), 6.922\left(\mathrm{~s}, 1 \mathrm{H}, \mathrm{C}_{4}-\mathrm{H}\right)$.
(6E)-Hydroximino-24-ethylcholest-4-en-3-one (1) The Jones' reagent of $0.5 \mathrm{~mL}(0.267 \mathrm{~mol} / \mathrm{L})$ was added dropwise to the solution of $8 \mathrm{a}(73 \mathrm{mg}, 0.164 \mathrm{mmol})$ in $10 \mathrm{~mL}$ of acetone in $10 \mathrm{~min}$. The reaction mixture was stirred at room temperature for $1 \mathrm{~h}$ and then neutralized with $10 \% \mathrm{~K}_{2} \mathrm{CO}_{3}$ solution. The suspension was poured over a silica gel column and eluted with ethyl acetate. The solvent was removed under reduced pressure. The residue was chromatographed on silica gel using petroleum ether $\left(60-90^{\circ} \mathrm{C}\right) /$ EtOAc $(3: 1)$ as eluent to give $46 \mathrm{mg}$ (63\%) of $1, \theta_{\mathrm{m} . p .} 197-198^{\circ} \mathrm{C}$; $\mathrm{IR}(\mathrm{KBr})$ v: 3383 , 2958, 2933, 2868, 1704, 1659, 1585, 1462, 1377, 1250, $984 \mathrm{~cm}^{-1}$; ${ }^{1} \mathrm{H} \mathrm{NMR}\left(\mathrm{CDCl}_{3}, 500 \mathrm{MHz}\right): 0.737$ (s, 3H, 18- $\left.\mathrm{CH}_{3}\right), 0.839$ (d, 3H, $J=6.5,26-\mathrm{CH}_{3}$ or $\left.27-\mathrm{CH}_{3}\right), 0.861\left(\mathrm{~d}, 3 \mathrm{H}, \mathrm{J}=6.5,26-\mathrm{CH}_{3}\right.$ or $27-$ $\left.\mathrm{CH}_{3}\right), 0.868$ (t, 3H, J = 8.5, 29- $\left.\mathrm{CH}_{3}\right), 0.956\left(\mathrm{~d}, 3 \mathrm{H}, \mathrm{J}=6.5,21-\mathrm{CH}_{3}\right)$, $1.064\left(\mathrm{~s}, 3 \mathrm{H}, 19-\mathrm{CH}_{3}\right), 2.274$ (ddd, $1 \mathrm{H}, \mathrm{J}=5.0,14.0,18.5, \mathrm{C}_{7}-$ $\alpha \mathrm{H}), 2.662$ (dd, $\left.1 \mathrm{H}, J=4.0,16.5, C_{2}-\beta \mathrm{H}\right), 3.097(\mathrm{dd}, 1 \mathrm{H}, J=3.5$, 18.5, $\mathrm{C}_{7}-\mathrm{BH}$ ), 6.778 (s, $1 \mathrm{H}, \mathrm{C}_{4}-\mathrm{H}$ ), 8.917 (brs, $\left.1 \mathrm{H},=\mathrm{N}-\mathrm{OH}\right) ;{ }^{13} \mathrm{C}$ $\mathrm{NMR}\left(\mathrm{CDCl}_{3}, 125 \mathrm{MHz}\right): 36.1$ (1-C), 33.4 (2-C), 200.9 (3-C), 126.3 (4-C), 155.8 (5-C), 149.2 (6-C), 26.0 (7-C), 33.4 (8-C), 50.0 (9-C), 42.5 (10-C), 21.3 (11-C), 39.3 (12-C), 46.0 (13-C), 56.7 (14-C), 24.0 (15-C), 28.0 (16-C), 55.9 (17-C), 11.9 (18-C), 18.9 (19-C), 38.9 (20C), 18.7 (21-C), 33.9 (22-C), 26.1 (23-C), 45.9 (24-C), 29.2 (25-C), 19.8 (26-C), 19.0 (27-C), 23.1 (28-C), 12.0 (29-C); LREIMS (70 eV, $\mathrm{m} / \mathrm{z} \%): 441\left(\mathrm{M}^{+}, 26\right), 426\left(\mathrm{M}^{+}-\mathrm{CH}_{3}, 100\right), 424\left(\mathrm{M}^{+}-\mathrm{OH}, 35\right), 152$ (38), 127 (89); HREIMS: $m / z 441.3604[M]^{+}$(calcd for $\mathrm{C}_{29} \mathrm{H}_{47} \mathrm{O}_{2} \mathrm{~N}_{1}$, 441.3601).

(6E)-Hydroximinocholest-4-en-3-one (2)

Yield 61\%, $\theta_{\text {m.p. }} 188-191^{\circ} \mathrm{C}$; IR(KBr) v: 3738, 3281, 2942, 2868, 1716, 1659, 1581, 1471, 1381, 1250, 1283, 1172, 1127, 1078, 1029, 980, 927, 866, 788, $735 \mathrm{~cm}^{-1} ;{ }^{1} \mathrm{H} \mathrm{NMR}\left(\mathrm{CDCl}_{3}, 500 \mathrm{MHz}\right): 0.734$ (s, $\left.3 \mathrm{H}, 18-\mathrm{CH}_{3}\right), 0.884\left(\mathrm{~d}, 3 \mathrm{H}, \mathrm{J}=6.2,26-\mathrm{CH}_{3}\right.$ or $\left.27-\mathrm{CH}_{3}\right), 0.897$ (d, $3 \mathrm{H}, J=6.2,26-\mathrm{CH}_{3}$ or $\left.27-\mathrm{CH}_{3}\right), 0.942\left(\mathrm{~d}, 3 \mathrm{H}, \mathrm{J}=6.5,21-\mathrm{CH}_{3}\right)$, $1.159\left(\mathrm{~s}, 3 \mathrm{H}, 19-\mathrm{CH}_{3}\right), 2.274$ (ddd, $1 \mathrm{H}, J=5.0,14.0,18.5, \mathrm{C}_{7}-\alpha \mathrm{H}$ ), 2.519 (dd, $\left.1 \mathrm{H}, J=5.1,14.7, \mathrm{C}_{2}-\beta \mathrm{H}\right), 3.437$ (dd, $1 \mathrm{H}, J=4.6,15.9, \mathrm{C}_{7}-$ $\beta \mathrm{H}), 6.338$ (s, $\left.1 \mathrm{H}, \mathrm{C}_{4}-\mathrm{H}\right), 9.033$ (brs, $\left.1 \mathrm{H}, \mathrm{N}-\mathrm{OH}\right) ;{ }^{13} \mathrm{C} \mathrm{NMR}\left(\mathrm{CDCl}_{3}\right.$, $125 \mathrm{MHz}$ ): 36.1 (1-C), 33.5 (2-C), 200.9 (3-C), 126.3 (4-C), 155.7 (5C), 149.2 (6-C), 28.0 (7-C), 33.3 (8-C), 50.0 (9-C), 42.5 (10-C), 21.3 (11-C), 39.5 (12-C), 46.0 (13-C), 56.7 (14-C), 24.0 (15-C), 28.1 (16C), 56.0 (17-C), 11.9 (18-C), 18.9 (19-C), 35.7 (20-C), 18.7 (21-C), 38.9 (22-C), 23.8 (23-C), 39.3 (24-C), 28.0 (25-C), 22.8 (26-C), 22.5 (27-C); LREIMS (70 eV, $\mathrm{m} / \mathrm{z} \%): 413\left(\mathrm{M}^{+}, 79\right), 396\left(\mathrm{M}^{+}-\mathrm{OH}, 100\right)$, $395\left(\mathrm{M}^{+}-\mathrm{H}_{2} \mathrm{O}, 30\right), 152(93)$; HREIMS: $\mathrm{m} / \mathrm{z} 413.3289$ [M] $^{+}$(calcd for $\mathrm{C}_{27} \mathrm{H}_{43} \mathrm{O}_{2} \mathrm{~N}_{1}, 413.3288$ ).

(4)

(6E)-Hydroximino-24-ethylcholest-4,22-dien-3-one

Yield $60 \%, \theta_{\text {m.p. }} 185-187^{\circ} \mathrm{C}$; $\operatorname{IR}(\mathrm{KBr})$ v: 3371, 2958, 2868, 1708, 1679, 1581, 1458, 1377, 1242, $976 \mathrm{~cm}^{-1} ;{ }^{1} \mathrm{H} \mathrm{NMR}\left(\mathrm{CDCl}_{3}\right.$, $500 \mathrm{MHz}$ ): 0.759 (s, 3H, 18- $\left.\mathrm{CH}_{3}\right), 0.833$ (t, 3H, J=7.5, 29- $\mathrm{CH}_{3}$ ), $0.826\left(\mathrm{~d}, 3 \mathrm{H}, \mathrm{J}=6.5,26-\mathrm{CH}_{3}\right.$ or $\left.27-\mathrm{CH}_{3}\right), 0.875(\mathrm{~d}, 3 \mathrm{H}, \mathrm{J}=6.5$, $26-\mathrm{CH}_{3}$ or $\left.27-\mathrm{CH}_{3}\right), 1.061$ (d, 3H, $\left.J=8.5,21-\mathrm{CH}_{3}\right), 1.069$ (s, 3H, $19-\mathrm{CH}_{3}$ ), 2.274 (ddd, $1 \mathrm{H}, J=5.5,14.5,18.0, \mathrm{C}_{7}-\alpha \mathrm{H}$ ), 2.657 (dd, $1 \mathrm{H}$, $\left.J=3.0,16.0, C_{2}-\beta H\right), 3.097$ (dd, $\left.1 H, J=5.0,18.0, C_{7}-\beta H\right), 5.065$ (dd, $\left.1 \mathrm{H}, J=9.0,15.5, \mathrm{C}_{22}-\mathrm{H}\right), 5.181$ (dd, $\left.1 \mathrm{H}, J=8.5,15.5, \mathrm{C}_{23}-\mathrm{H}\right), 6.779$ (s, $\left.1 \mathrm{H}, \mathrm{C}_{4}-\mathrm{H}\right) ;{ }^{13} \mathrm{C} \mathrm{NMR}\left(\mathrm{CDCl}_{3}, 125 \mathrm{MHz}\right) \delta: 38.9$ (1-C), 33.5 (2-C), 200.9 (3-C), 126.3 (4-C), 155.8 (5-C), 149.1 (6-C), 28.7 (7-C), 31.9 (8-C), 51.3 (9-C), 42.4 (10-C), 21.3 (11-C), 39.2 (12-C), 46.0 (13-C), 56.8 (14-C), 24.1 (15-C), 31.8 (16-C), 55.9 (17-C), 12.2 (18-C), 18.9 (19-C), 40.4 (20-C), 21.1 (21-C), 137.9 (22-C), 129.7 (23-C), 50.1 
(24-C), 33.3 (25-C), 21.2 (26-C), 18.7 (27-C), 25.4 (28-C), 12.1 (29C); LREIMS (70 eV, $\mathrm{m} / \mathrm{z} \%): 439\left(\mathrm{M}^{+}, 100\right), 422\left(\mathrm{M}^{+}-\mathrm{OH}, 45\right), 396$ $\left(\mathrm{M}^{+}-\mathrm{CH}\left(\mathrm{CH}_{3}\right)_{2}, 89\right), 298(59), 152(87)$; HREIMS: $\mathrm{m} / \mathrm{z} 439.3445[\mathrm{M}]^{+}$ (calcd for $\mathrm{C}_{29} \mathrm{H}_{45} \mathrm{O}_{2} \mathrm{~N}_{1}, 439.3445$ ).

The similar method was used for synthesizing the compounds 2 and 4 . Therefore, only experimental details for the synthesis of compound 1 are reported.

\section{Antiproliferative activity}

\section{Materials and methods}

Stock solutions of compounds 1, 2 and 4, were prepared in sterile dimethyl sulfoxide (DMSO) (Sigma) at a concentration of $10 \mathrm{mg} / \mathrm{mL}$ and afterwards diluted with complete nutrient medium (RPMI-1640) supplemented with 10\% heat inactivated fetal bovine serum and $0.1 \mathrm{~g} / \mathrm{L}$ penicillin $\mathrm{G}+0.1 \mathrm{~g} / \mathrm{L}$ streptomycin sulfate.

\section{Cell culture}

Sk-Hep-1, H-292, PC-3 (ATCC) and Hey-1B (a gift from Dr. Yan $\mathrm{Xu}$, University of Indiana) cells were cultured in a proper medium supplemented with $10 \%$ fetal bovine serum in a humidified atmosphere of $5 \% \mathrm{CO}_{2}$ at $37^{\circ} \mathrm{C}$.

\section{Treatment of cancer cells}

Cancer cells $\left(4 \times 10^{3}\right.$ cells $\left./ 200 \mu \mathrm{L}\right)$ were seeded into each well of a 96 -well microtiter plate. After incubation for $24 \mathrm{~h}$, the compounds with a series of concentrations (range $20-80 \mu \mathrm{g} / \mathrm{mL}$ ) were added to the cells. An equal amount of DMSO was added to the cells used as negative controls. All were treated in triplicate.

\section{Determination of cell viability}

MT Stetrazolium salt (3-(4,5-dimethylthiazol-2-yl)-5-(3carboxymethoxyphenyl)-2-(4-sulfophenyl)-2H-tetrazolium) (CellTiter 96 AQueous Non-Radioactive Cell Proliferation Assay, Cat.\# G5421, Promega Corporation) dye reduction assay was used. The assay is dependent on the MTS being reduced to an aqueous, soluble formazan by dehydrogenase enzymes found in metabolically active cells. The quantity of formazan product as measured by the amount of $490 \mathrm{~nm}$ absorbance is directly proportional to the number of living cells in culture. Briefly, after treatment (see Section2.2.3) for $72 \mathrm{~h}$, the medium was removed and the cells were incubated with $100 \mu \mathrm{L}$ of fresh medium plus $20 \mu \mathrm{L}$ of MTS solution according to the instruction for additional $4 \mathrm{~h}$. The absorbance (A) at $490 \mathrm{~nm}$ was measured using an Beckman coulter LD400 AD/LD analysis spectrometer. IC $_{50}$ concentration was defined as the concentration of an agent inhibiting cell survival by $50 \%$, compared to a control.

\section{Results and discussion}

The initial studies for the synthesis of this kind of oximesteroid system were based on the methodology developed by Holland et al. [8]. Seven steps were needed to synthesize compound 1 as reported in reference [4]. Later Kovganko et al. reported two new synthesis routes for the compound 1, both were rather complicated and the overall yields were relatively low $[9,10]$. Here, we introduce a new synthetic method for the steroidal oxime compound 1, 2 and 4 with higher overall yields and fewer synthetic steps. For example, using $\beta$-sitosterol as raw material, four steps are needed for synthesizing the compound 1 with an overall yield of 33\%. The synthesis route of the compound 1, 2 and 4 is as shown in Fig. 1.<smiles>[R]C(C)[C@H]1CC[C@H]2[C@@H]3CCC4=CC(O)CC[C@]4(C)[C@H]3CC[C@@]21C</smiles>

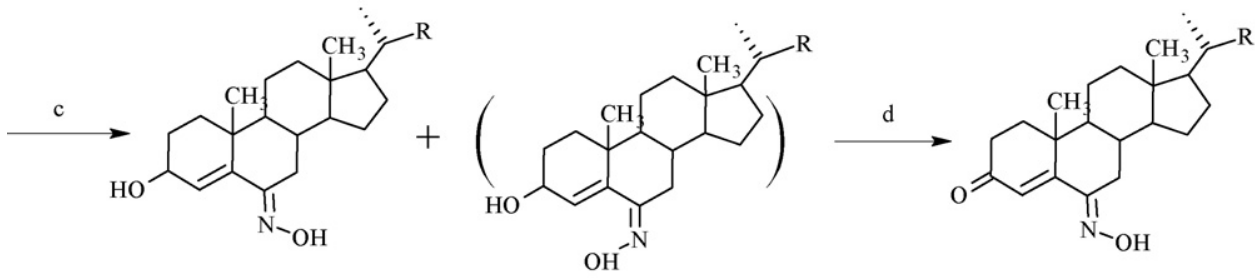

8

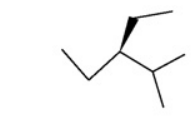

$\mathrm{R}=\mathrm{a}$
9

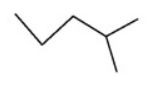

b
$1(\mathrm{R}=\mathrm{a}), \mathbf{2}(\mathrm{R}=\mathrm{b}), \mathbf{4}(\mathrm{R}=\mathrm{c})$

Fig. 1 - The synthesis of some (6E)-hydroximino-4-en-3-one steroids. (a) PCC/C H2Cl2; (b) NaBH4/CH3OH, CoCl2.6H2O; (c) NaAc.3H2O/95\% C2H5OH, H2OH.HCl; (d) Jones agent/acetone. 
Table 1 - In vitro antitumor activities $\left(\mathrm{IC}_{50} \mu \mathrm{g} / \mathrm{mL}\right)$ of 1,2 and $4^{\mathrm{a}}$

\begin{tabular}{lcccc} 
Compound & Sk-Hep-1 & H-292 & PC-3 & Hey-1B \\
\hline 1 & $\geq 80$ & $\geq 80$ & $\geq 80$ & $\geq 80$ \\
2 & 33 & 32.6 & 35 & 54 \\
4 & 43 & 59.5 & 44 & 37 \\
\hline
\end{tabular}

${ }^{a}$ MTS method was used to assay of antiproliferative activity. biological activity. This is consist with the conclusion obtained by Rodríguez et al. [7].

\section{Acknowledgments}

The authors acknowledge the financial support of the National Natural Science Foundation of China (Project: 20562001), the Natural Science Foundation of Guangxi Province (Guike: 057554) and the graduate innovation fund of Guangxi Province.

\section{REFERENCES}

In step1, $\beta$-sitosterol (5a) is transformed into the corresponding 24-ethylcholest-4-en-3,6-dione (6a) via oxidation with PCC in $\mathrm{CH}_{2} \mathrm{Cl}_{2}$ in $86 \%$ yield. Selective reduction of $6 \mathrm{a}$ by $\mathrm{NaBH}_{4}$ in the presence of $\mathrm{CoCl}_{2}$ gives 24-ethylcholest-4-en-3 $\beta$ ol-6-one (7a) in $85 \%$ yield according to the synthetic method we developed [11]. The structure of 7a was confirmed by comparing IR and ${ }^{1} \mathrm{H}$ NMR spectra with those of the analogous compound that was synthesized previously in Ref. [12].

Next, the oxime $8 \mathrm{a}$ is obtained by the reaction of $7 \mathrm{a}$ with hydroxylamine hydrochloride in ethanol in the presence of $\mathrm{NaOAc}$ in $75 \%$ yield. The structure of $8 \mathrm{a}$ was proved by spectral data. At the same time, cis-isomer $9 \mathrm{a}$ of $8 \mathrm{a}$ was obtained in $9.5 \%$ yield.

Oxidation of $8 \mathrm{a}$ by Jones' reagent in acetone gives the target steroid 1 in $69 \%$ yield. The IR and ${ }^{1} \mathrm{H}$ NMR spectra data of 1 are perfectly consistent with those of the natural compound 1 .

The compound 2 and 4 were prepared in the similar synthetic method to the compound 1.

To determine the biological activity of these compounds, we investigated their ability against four human tumor cell lines: Sk-Hep-1 (human liver carcinoma), H-292 (human lung carcinoma), PC-3 (human prostate carcinoma) and Hey-1B (human ovarian carcinoma). The results, expressed as $\mathrm{IC}_{50}$ values in $\mu \mathrm{g} / \mathrm{mL}$, are reported in Table 1.

Our results showing in the Table 1 revealed that the compound 2 and 4 displayed a modest cytotoxic activity against these cancer cells. Interestingly the structure of side chains on these steroidal oximes plays an important role in their cytotoxicity. The antineoplastic activity of these compounds increases along with the order of the side chain attached: cholesterol-like side chain(2) > stigmasterollike side chain(4)>sitosterol-like side chain(1). The presence of a cholesterol-type side chain appears to be necessary for the
[1] Blunt JW, Copp BR, Hu WP, Munro MHG, Northcote PT, Prinsep MP. Marine natural products. Nat Prod Rep

[2] Blunt JW, Copp BR, Munro MHG, Northcote PT, Prinsep MP. Marine natural products. Nat Prod Rep 2005;22:15-61.

[3] Faulkner DJ. Marine natural product. Nat Prod Rep 2001;18:1-49.

[4] Jaime R, Lucia N, Solange P, Carlos J. Isolation and synthesis of the first natural 6-hydroximino- 4-en-3-one steroids from the sponges Cinachyrella spp. Tetrahedron Lett 1997;38:1833.

[5] Xiao DJ, Peng XD, Deng SZ. Structure elucidation of (3E)-cholest-4-en-3,6-dione-3-oxime in marine sponge Cinachyrella australiensis from the south china sea. Chin J of Org Chem 2005;25(12):1606-9.

[6] Zeelen FJ. Medicinal chemistry of steroids. Amsterdam: Elsevier Sci. Publ.; 1990. p. 357. $6 E$-hydroximino-4-ene steroids: structure/activity studies. J Med Chem 2001;44:2612-8.

[8] Holland HL, Kumaresan S, Tan L, Njar VCO. Synthesis of new 6-hydroximino- oxo steroids, a new class of aromatase inhibitor. J Chem Soc Perkin Trans 1992;1:585-7.

[9] Kovganko NB, Chernov YuG. Novel synthesis of (24R, 6E)-24-ethylcholest-6-hydroxyimno-4-en-3-one, a steroidal oxime from Cinachyrella spp. sponges. Chem Nat Compd 2000;36(2):189.

[10] Kovganko NV, Chernenko Yu G. Synthesis of oxime of stigmastane $5 \beta$-hydroxy-6-ketosteroids. Chem Nat Compd 2001;37(3):256.

[11] Jianguo Cui, Longmei Zeng, Jingyu Su. Synthesis of some polyhydroxysterols and investigation of relationship between their structure and cytotoxicity. Chem J Chin Univ (Chinese) 2000;21(9):1399-404.

[12] Jianguo Cui, Longmei Zeng, Jingyu Su, Cuiwu Lin. Regio- and stereo-selective reductions of steroidal 4-en-3,6-dione. Chem Res Chin Univ 2002;18(4):400-4. 2007;24:31-86

[7] Deive N, Rodríguez J, Jiménez C. Synthesis of cytotoxic 